schen Leistung (insbesondere: Präsenzpflichten). Angehängt sind Hinweise zur Behandlungspflicht, Sorgfaltspflicht, zu den Vertretungsmöglichkeiten, der Privatliquidation bei PKV-Patienten und der Vergütung neuer Leistungen.

Bei der Darstellung der berufsrechtlichen Pflichten liegt das Schwergewicht bei der Dokumentationspflicht, den Kooperationsmöglichkeiten, dem Einsichtsrecht der Patienten und den Gutachtenpflichten. Aktuell sind die Hinweise zu den Sorgfaltspflichten bei der sog. „Internetpsychotherapie“. In einem letzten Abschnitt geht Stellpflug auch auf die Möglichkeiten und Grenzen einer Kostenerstattung für Psychotherapeuten nach $₫ 13$ Abs. 3 SGB V ein.

Das Literaturverzeichnis ist umfangreich, kann jedoch nicht als aktuell bezeichnet werden (bei Rieger beispielsweise ist statt des aktuellen „Heidelberger Kommentars“ noch das „Lexikon des Arztrechts“ aufgeführt). Im Hinblick auf den Leserkreis wird in den Fußnoten an den einschlägigen Stellen nicht durchgehend auf weiterführende juristische Literatur und Rechtsprechung verwiesen; wer Einzelfragen vertieft behandeln möchte, findet im Literaturverzeichnis und anderen medizinrechtlichen Handbüchern (mittlerweile zahlreich) ausreichend Hinweise. Der Text ist gegenüber der 1. Auflage deutlich umfangreicher; auf die Darstellung von Normen wurde verzichtet.

Insgesamt: Eine erfreulich klare Darstellung zu einem Thema, das in anderen Handbüchern zum Vertragsarztrecht nicht durchgehend ausführlich dargestellt wird, das aber mittlerweile zunehmend an Bedeutung gewonnen hat.

\section{DOI: 10.1007/s00350-013-3617-8}

\section{Arzthaftungsrecht. Leitfaden für die Praxis.}

Von Wolfgang Frahm, Wolfgang Nixdorf und Alexander Walter. Verlag Versicherungswirtschaft, 5. Aufl. Karlsruhe 2013, XX u. 319 S. mit 1 CD-ROM, kart., $€ 68,00$

Nach vier Jahren erscheint die Neuauflage des Buches ,Arzthaftungsrecht" von Wolfgang Frahm, Vorsitzender Richter am OLG Schleswig, und Alexander Walter, Richter am LG Koblenz (zur Vorauflage s. die Rezension von Achterfeld, MedR 2009, 505). Der Praxisleitfaden soll bei der alltäglichen Bearbeitung einschlägiger Schadensfälle Hilfe leisten, er richtet sich in erster Linie an Rechtsanwälte und Richter sowie Mitarbeiter von Versicherungsunternehmen, Verbänden und Krankenhäusern. Die Verfasser bieten eine aktuelle Darstellung der in Rechtsprechung und Schrifttum ständig fortentwickelten Grundfragen und Zusammenhänge des Arzthaftungsrechts. Das Werk is gegenüber der Vorauflage um sechzig Seiten angewachsen. Dabe wurde die Randnummernzählung erfreulicherweise beibehalten, wo erforderlich um Buchstaben ergänzt.

Untergliedert ist die Schrift in sechs Teile. Der erste (S. 1-64) gilt den Haftungsgrundlagen aus Vertrag, GoA und Delikt. Eingehend werden die unterschiedlichen Vertragskonstellationen in Einzelund Gruppenpraxis, bei Zusammenarbeit und Überweisung sowie bei Krankenhausbehandlungen dargestellt. Daran schließt der zweite Teil an zu Haftenden und Haftung für Hilfspersonen (S. 65-84). Der dritte Teil zum Behandlungsfehler ist der umfangreichste (S. 85-199), thematisiert werden u. a. auch Kostendruck und Standard, haftungsrechtliche Bedeutung von Richtlinien und Leitlinien, ärztliche Therapiefreiheit sowie Fragen der Beweisführung und Beweislast, auf Rechtsfolgenseite das Sonderproblem der Familienplanungsschäden.

Prof. Dr. iur. Christian Katzenmeier,

Universität zu Köln, Deutschland
Im vierten Teil wird die facettenreiche Rechtsprechung zur ärztlichen Aufklärungspflicht präsentiert (S. 201-274), Teil fünf gilt der Verjährung (S. 249-274), der sechste und letzte Teil verfahrensrechtlichen Fragen (S. 275-310).

Das Patientenrechtegesetz haben Frahm/Walter bereits umfassend eingearbeitet. Sie zeigen auf, dass die $\$ \$ 630$ aff. BGB einige neue Verpflichtungen statuieren, mitunter Vorgaben der Rechtsprechung verschärft werden und teilweise die Gefahr von Missverständnissen besteht ,aufgrund der verkürzten gesetzlichen Regelung der richterrechtlichen Grundsätze, regelungstechnischer Irritationen und einer unbestimmten bzw. ungenauen Gesetzesbegründung" (S. 3).

Differenziert erörtert wird etwa die wirtschaftliche Informationspflicht nach $\$ 630$ c Abs. 3 BGB (S. 46 ff.) oder die Fehleroffenbarungspflicht gem. $\$ 630 \mathrm{c}$ Abs. 2 S. 2 BGB (S. 50 ff.). In der grundsätzlichen Verpflichtung des Arztes auf die allgemein anerkannten Standards gem. \$630a Abs. 2 BGB erkennen die Verfasser keine Gefahr für die Freiheit der Methodenwahl (S. 123 ff.). Die im Schrifttum kritisierte Doppelung der Aufklärungs- und Informationspflichten in $\iint 630$ c Abs. 2 S. 1, 630e BGB erachten sie als unproblematisch (S. 127). Bei der Kodifizierung der Beweislastumkehr wegen eines groben Behandlungsfehlers in $\$ 630 \mathrm{~h}$ Abs. $5 \mathrm{~S} .1 \mathrm{BGB}$ machen sie Flüchtigkeitsfehler des Gesetzgebers aus (S. 148f., Fn. 287 u. 290), die Begründung zu $\$ 630 \mathrm{~h}$ Abs. 5 S. 2 BGB wird mit Grund als erstaunlich knapp (S. 152), die zur Dokumentationspflicht in $\$ 630 \mathrm{f}$ BGB in puncto „faktische Beweissicherung“ zu Recht als missverständlich bezeichnet (S. 157). Auf das neue Erfordernis der Revisionssicherheit einer EDV-Dokumentation nach $\$ 630$ f Abs. 1 S. 3 BGB wird hingewiesen (S. 162f.), ebenso auf die Ausweitung des Einsichtsrechts durch die Regelung in $\$ 630 \mathrm{~g} \mathrm{BGB}$ (S. $167 \mathrm{ff}$.). Näher analysiert wird die Beweislastregelung bei Verwirklichung eines voll beherrschbaren Risikos, \$630h Abs. 1 BGB (S. 174ff.), die Ausführungen zum geltenden Beweismaß sind demgegenüber knapp (S. 189f.). Verfasser stellen klar, dass angesichts der Regelung in $\$ 630$ e Abs. $2 \mathrm{~S} .1 \mathrm{Nr} .1$ BGB eine Formularaufklärung auch bei Reihenbehandlungen nich ausreicht (S. 213) und dass eine Delegation der Aufklärung auf nichtärztliches Hilfspersonal nach $\$ 630$ e Abs. 2 S. 1 Nr. 1 BGB weiterhin unzulässig ist (S. 232). Insgesamt merken Frahm/Walter an, dass der bislang von der Rechtsprechung stets betonte Gleichlauf von vertraglicher und deliktischer Haftung schwieriger wird, wo die $\$ \$ 630 \mathrm{aff}$ BGB konkret ausgestaltete Verpflichtungen vorgeben (S. 86f.), und stellen die Frage, ob die Kodifikation der Beweisregeln ein Hemmnis für die Fortentwicklung des Rechts bedeutet (S. 137f.). Die Ausführungen zu den neuen BGB-Vorschriften sind sehr gut in den Text integriert, die Analysen sind ausnahmslos scharfsinnig und fundiert, liefern wichtige Impulse für die künftige Auslegung und Anwendung in der Rechtspraxis.

Besondere Erwähnung verdient schließlich, dass die beiden Richter nicht nur die reichhaltige Judikatur, sondern ebenso das umfangreiche Schrifttum sichten, auswerten und gewissenhaft nachweisen. So ist die Schrift weit mehr als ein reiner Rechtsprechungsspiegel oder Praxisleitfaden, das Werk ist vielmehr zugleich ein Lehrbuch zum Arzthaftungsrecht. Im Ergebnis weichen die Verfasser allerdings in keinem Punkt von der höchstrichterlichen Rechtsprechung $\mathrm{ab}$, folgen dem BGH vielmehr ausnahmslos, und zwar bei streitigen Grundsatzfragen (zur Körperverletzungsdoktrin s. auf S. 55 f.) wie in zweifelhaften Einzelpunkten (s. etwa zur Aufklärungspflicht über Risiken im Promillebereich auf S. 215, zu den sehr weit reichenden Organisationspflichten auf S. 106 ff., zum Beginn der Verjährung des Regressanspruchs eines Sozialversicherungsträgers auf S. 261 ff.).

Fazit: Wolfgang Frahm und Alexander Walter legen eine sehr sorgfältig überarbeitete, aktualisierte und fortgeschriebene Neuauflage ihres bewährten Werkes vor und lösen die hohen Erwartungen ein. Das Buch ist dogmatisch anspruchsvoll, praxisrelevant, sehr klar verständlich geschrieben, mithin uneingeschränkt und mit Nachdruck jedem am Arzthaftungsrecht Interessierten zur Lektüre zu empfehlen. 